\title{
ON THE ZEROS OF DIRICHLET $L$-FUNCTIONS. III
}

BY

\section{AKIO FUJII( $\left.{ }^{1}\right)$}

ABSTRACT. It is shown that the ordinates of the zeros of the Riemann zeta function are uniformly distributed. Similar results pertain to zeros of $L$-functions.

1. Introduction. In an earlier work [2], we established results which measure the irregularity of the distribution of the zeros of the zeta function. Here we use the previous results to show that the zeros of the zeta function are uniformly distributed. Let $\rho=\beta+i \gamma$ denote the generic nontrivial zero of the zeta function, and let $N(T)$ denote the number of these zeros for which $0<\gamma$ $\leqslant T$. For $T \geqslant 10,0 \leqslant h \leqslant T, 0 \leqslant \alpha \leqslant 1$, let $A_{T}(\alpha, h)$ denote the number of zeros $\rho, 0<\gamma \leqslant T$, for which $0 \leqslant\{\gamma / h\} \leqslant \alpha$. Here $\{\theta\}$ is the fractional part of $\theta,\{\theta\}=\theta-[\theta]$. To show that the ordinates $\gamma$ are uniformly distributed modulo $h$ we seek bounds for the discrepancy

$$
D_{T}(h)=\sup _{0 \leqslant \alpha<1}\left|A_{T}(\alpha, h) / N(T)-\alpha\right| .
$$

THEOREM. For $\epsilon>0, T>T_{0}(\epsilon),(\log T)^{-1} \leqslant h \leqslant T^{1 / 3}$, we have, in the above notation,

$$
D_{T}(h)<<(h \log T)^{-1+\epsilon}
$$

A sequence is said to be uniformly distributed if the discrepancy of the first $n$ terms tends to zero as $n$ tends to infinity. Thus the numbers $\gamma$ are uniformly distributed $(\bmod h)$, and uniformly so for $H(T) \leqslant h \leqslant T^{1 / 3}$, provided that $H(T) \log T \rightarrow \infty$. Elliott [1] has shown that $D_{T}(h)=o(1)$ for $h$ fixed, but his method does not provide as precise estimates as we obtain. Our main interest is in the small values of $h$. Trivial arguments show that $D_{T}(h)=o(1)$ for $T^{1 / 3} \leqslant h=o(T)$, but the quantitative estimate for $D_{T}(h)$ becomes weaker for these larger $h$ due to the gradual increase in the density of zeros as $t$ increases. We conjecture that $D_{T}(h)=o(1)$ even when $h=c(\log T)^{-1}$, but present methods fail to provide such delicate results.

Received by the editors April 8, 1974.

AMS (MOS) subject classifications (1970). Primary 10H05, $10 \mathrm{H} 10$. of zeros.

Key words and phrases. Riemann zeta function, Dirichlet $L$-functions, distribtuion

(1) Supported in part by NSF grant GP $36418 \times 1$. The author is grateful to the referee who has kindly rewritten the manuscript in a very neat form for publication. Thanks are also due to Professor Patrick Gallagher. 
The estimate of the Theorem can be extended to hold uniformly for zeros of an $L$-function $(\bmod q)$, for $q<T^{1 / 4-\delta}$, and also for zeros of $\zeta(s)$ in a short interval, $T<\gamma<T+H$, for $H>T^{1 / 2+\delta}$.

2. Proof of the Theorem. As usual, we let $S(t)=\pi^{-1}$ arg $\zeta(1 / 2+i t)$. The Riemann-von Mangoldt formula (see [3, p. 179]) for $N(t)$ asserts that

$$
N(t)=(t / 2 \pi) \log (t / 2 \pi)-t / 2 \pi+7 / 8+R(t)
$$

for $t>0$, where $R(t)=S(t)+O(1 / t)$. Thus to estimate $N(t+h)-N(t)$ we require an estimate of $S(t+h)-S(t)$. To this end we quote from [2] the following

LemmA. Let $h \leqslant T^{1 / 3}, T \geqslant 10$. Then for all integral $k \geqslant 1$,

$$
\begin{aligned}
\int_{0}^{T}(S(t+h)-S(t))^{2 k} d t= & C_{k} T(2 \log (3+h \log T))^{k} \\
& \cdot\left(1+O\left((A k)^{3 k}(\log (3+h \log T))^{-1 / 2}\right)\right),
\end{aligned}
$$

where $C_{k}=(2 k) !(2 \pi)^{-2 k} k !^{-1}$.

For brevity we let $\delta=(\log T)^{-1}$. If $0 \leqslant \alpha \leqslant 1 / 2 \delta h^{-1}$, then

$$
\left|A_{T}(\alpha, h) / N(T)-\alpha\right| \leqslant\left|A_{T}\left(1 / 2 \delta h^{-1}, h\right) / N(T)-1 / 2 \delta h^{-1}\right|+1 / 2 \delta h^{-1} .
$$

A similar inequality applies if $1-1 / 2 \delta h^{-1} \leqslant \alpha \leqslant 1$, so it suffices to show that

$$
\left|A_{T}(\alpha, h) / N(T)-\alpha\right|<<(h \log T)^{-1+\epsilon}
$$

for $1 / 2 \delta h^{-1} \leqslant \alpha \leqslant 1-1 / 2 \delta h^{-1}$.

Let $\varphi_{+}(t)$ be the piecewise linear function with period $h$, determined by the vertices $(-\delta, 0),(0,1),(\alpha h, 1),(\alpha h+\delta, 0)$; similarly let $\varphi_{-}(t)$ be determined by the vertices $(0,0),(\delta, 1),(\alpha h-\delta, 1),(\alpha h, 0)$. Then

By (1),

$$
\sum_{0<\gamma \leqslant T} \varphi_{-}(\gamma) \leqslant A_{T}(\alpha, h) \leqslant \sum_{0<\gamma \leqslant T} \varphi_{+}(\gamma)
$$

$$
\begin{aligned}
\sum_{0<\gamma<T} \varphi_{-}(\gamma) & =\int_{0}^{T} \varphi_{-}(t) d N(t) \\
& =\frac{1}{2 \pi} \int_{0}^{T} \varphi_{-}(t) \log \frac{t}{2 \pi} d t+\int_{0}^{T} \varphi_{-}(t) d R(t) .
\end{aligned}
$$

Now

so

$$
0<\log (t / 2 \pi)-\log (m h / 2 \pi)<1 / m \text { for } m h \leqslant t \leqslant(m+1) h \text {, }
$$

$$
\int_{m h}^{(m+1) h} \varphi_{-}(t) \log \frac{t}{2 \pi} d t=\left(\alpha-\delta h^{-1}\right) \int_{m h}^{(m+1) h} \log \frac{t}{2 \pi} d t+O(h / m)
$$


for $m \geqslant 1$. Thus the first integral in (3) is

$$
\begin{aligned}
& =\left(\alpha-\delta h^{-1}\right)((T / 2 \pi) \log (T / 2 \pi)-T / 2 \pi)+O(h \log T) \\
& =N(T)\left(\alpha+O\left(\delta h^{-1}\right)+O\left(h T^{-1}\right)\right) .
\end{aligned}
$$

On the other hand, $S(t)<<\log t$, so the second integral in (3) is

$$
\begin{aligned}
& =\int_{0}^{T} R(t) d \varphi_{-}(t)+O(\log T) \\
& =-\delta^{-1} \int_{0}^{T}(S(t+\alpha h-\delta)-S(t)) \psi(t) d t+O(\log T),
\end{aligned}
$$

where $\psi(t)$ is piecewise constant with period $h, \psi(t)=1$ for $0<t \leqslant \delta, \psi(t)=0$ for $\delta<t \leqslant h$. By Hölder's inequality the integral above is

$$
\leqslant\left(\int_{0}^{T}|\psi(t)|^{2 k /(2 k-1)} d t\right)^{1-1 / 2 k}\left(\int_{0}^{T}(S(t+\alpha h-\delta)-S(t))^{2 k} d t\right)^{1 / 2 k} .
$$

By the Lemma this is

$$
<_{k}\left(\delta h^{-1} T\right)^{1-1 / 2 k} T^{1 / 2 k}(\log (2 h \log T))^{1 / 2} .
$$

Thus

$$
\int_{0}^{T} \varphi_{-}(t) d R(t) \ll<_{k} N(T)(h \log T)^{-1+1 / 2 k}(\log (2 h \log T))^{1 / 2} .
$$

Combining this with (4), we find that

$$
\sum_{0<\gamma<T} \varphi_{-}(\gamma)=\left(\alpha+O(h \log T)^{-1+\epsilon}\right) N(T)
$$

for $T>T_{0}(\epsilon)$. A similar argument applies to $\varphi_{+}$, so the-Theorem now follows from (2).

\section{BIBLIOGRAPHY}

1. P. D. T. A. Elliott, The Riemann zeta function and coin tossing, J. Reine Angew. Math. 254 (1972), 100-109. MR 47 \#1761.

2. A. Fujii, On the zeros of Dirichlet L-functions. I, Trans. Amer. Math. Soc. 196 (1974), 225-235. MR 50 \#2096.

3. E. C. Titchmarsh, The theory of the Riemann zeta function, 2 nd ed., Clarendon Press, Oxford, 1951. MR 13, 741.

SCHOOL OF MATHEMATICS, INSTITUTE FOR ADVANCED STUDY, PRINCETON, NEW JERSEY 08540

Current address: Department of Mathematics, Rikkyo University, Tokyo, Japan 